This document is published in:

Ravenscroft, A. et al. (eds.) (2012). 7th European Conference of Technology Enhanced Learning, EC-TEL 2012, Saarbrücken, Germany, September 18-21, 2012. Proceedings. Part II (Lecture Notes in Computer Science, 7563), Springer, pp. 113-125.

DOI: 10.1007/978-3-642-33263-0_10

(c) 2012 Springer-Verlag Berlin Heidelberg 


\title{
Enhancing Orchestration of Lab Sessions by Means of Awareness Mechanisms
}

\author{
Israel Gutiérrez Rojas ${ }^{1,2}$, Raquel M. Crespo García ${ }^{1}$, and Carlos Delgado Kloos ${ }^{1}$ \\ ${ }^{1}$ Universidad Carlos III de Madrid, Avda. Universidad 30, E-28911 Leganés, Spain \\ ${ }^{2}$ Institute IMDEA Networks, Avda. del Mar Mediterráneo 22, E-28918 Leganés, Spain \\ \{igrojas, rcrespo, cdk\} @it.uc3m.es
}

\begin{abstract}
Orchestrating learning is a quite complex task. In fact, it has been identified as one of the grand challenges in Technology Enhanced Learning (TEL) by the Stellar Network of Excellence. The objective of this article is to provide teachers and students with a tool to help them in their effort of orchestrating learning, that makes use of awareness artefacts. Using this powerful mechanism in lab sessions, we propose four different aspects of orchestration as the target for improvement: the management of the resources in the learning environment; the interventions of the teacher and provision of formative feedback; the collection of evidences for summative assessment; and the re-design of the activity, adjusting some parameters for future enactments. The proposal has been tested in a real course of Multimedia Applications with junior students (3rd course), measuring the benefits for the orchestration.
\end{abstract}

Keywords: Orchestration, awareness, lab session, problem-based learning, formative assessment.

\section{Introduction}

Many Higher Education courses, ranging from engineering to social sciences, have a practical component; that is, the structure of the course is composed by theoretical sessions (lectures) and practical sessions in the computer room, where the students have a computer available to work in the proposed hands-on activity. These face-toface sessions at the computer lab (henceforth called lab sessions) have a common structure: the teacher proposes a practical task (or set of tasks) to the students; the students work on the proposed task by themselves in their computer; when they encounter a difficulty that cannot overcome by themselves, they raise hand in order to indicate the teacher they have a question; the teacher moves around the room solving questions of the students who raised hand. There are other aspects of the lab session that are specific to the activity in particular: the students work in the computer individually, in pairs or in a group (individual/collaborative activity), there could be one or several teachers (or teaching assistants), the development of the activity could have implications for summative assessment or just have a pure formative component, etc.

In some countries, the education budget has been cut out consequence an increase of the students/teacher ratio in lab sessions. When the ratio is higher than 20 , several 
problems emerge, intrinsically related to the orchestration of the lab session: the teacher is not able to provide feedback to the students at the same rate that new questions appear; due to the scarcity of the teacher resource, the students compete for the attention of the teacher (e.g., they stand up and wait near to the teacher while she is attending other students, and when she finishes the explanation they grab her attention to help them); the order in which the teacher provide feedback to the students is unfair, regarding parameters like waiting time of the students or progress in the assignment; the teacher has not enough time to check the progress of all the students during the session, mainly the ones who did not ask for help.

In order to mitigate these problems, a tool has been designed that provides teachers with awareness mechanisms in lab sessions. Using the awareness information, teachers gain knowledge about the state of the class: progress of all the students, students who asked for help and when, etc. And they are able to enhance the orchestration of the lab session in several ways: the management of the resources in the learning environment (e.g., feedback time); the interventions and provision of formative feedback; the collection of evidences for summative assessment; and the redesign of the activity, adjusting some parameters for future enactments.

The rest of this article is structured as follows: the next section introduces relevant research about orchestrating learning and awareness; section 3 will be devoted to defining the proposed tool that provides awareness mechanisms to the teacher; in section 4 a validation of such a tool will be presented, based on an experiment in a real setting; finally, in section 5 the conclusions of this work are described as well as some lines of future work related to them.

\section{Relevant Literature}

Orchestrating learning is a quite complex task. In fact, it has been identified as one of the grand challenges in Technology Enhanced Learning (TEL) by the Stellar Network of Excellence [1]. Moreover, the concept of "orchestrating learning" has different definitions in the TEL community and therefore a different meaning depending of the authors of a publication. In [2], a literature review of orchestrating learning in TEL is carried out; emerging from the review, a conceptual framework is defined consisting of $5+3$ aspects of orchestration: 5 aspects about what orchestration is and 3 aspects about how orchestration has to be implemented. Regarding the orchestration definition, the aspects described were (1) design/planning of the learning activities, (2) regulation/management of these activities, (3) adaptation/flexibility/intervention (adaptation of the learning flow to emergent events), (4) awareness/assessment of what happens in the learning process and (5) the different roles of the teacher and other actors. Regarding how the orchestration should be done, (a) pragmatism/practice as opposed to TEL-expert, (b) alignment/synergy to the intended learning outcomes and (c) models/theories that guide the learning orchestration, were the identified aspects. In this work, we have made use of the 5+3 aspects framework in order to structure the contributions to enhance the orchestration by means of awareness mechanisms. 
The concept of awareness in the field of Computer Supported Collaborative Work (CSCW) refers to exchange of information among several workers that work on a collaborative activity, regarding status, activity and availability. In this work, we apply these same principles to the context of teaching and learning, considering the awareness as a mechanism that could be used to deal with a complex learning scenario built over different orchestration aspects because it permits teachers and students to get to know better these aspects.

In [3], Alavi et at. use the concept of awareness in the same way in the context of technology-enhanced learning. They analyse the interactions between teacher assistants and learners in recitations sections (sessions of problem-based activities with teaching assistants), and make use of lamps as distributed awareness mechanisms. The lamps are used by students in order to indicate progress (lamp colour) and to request feedback from the teachers (lamp blinking). While the interactions in a recitation section are quite similar to those in lab sessions, both works take a very different approach to overcome the same orchestrations problems: in Alavi's work they focus on using Human Computer Interaction (HCI) aspects (e.g., ambient displays for distributed awareness); instead, our work stresses the importance of recording students' traces and process them to create information useful for the teacher. Therefore, both approaches have advantages: in Alavi's work, the groups of students are aware of their colleagues progress and problems; in our work, the information is processed to offer the teacher a personalised view of the interaction data, and the information could be used after the class to review the session (note: in the context of our work, it is assumed that the assignments are delivered to the students in the form of a web page, which the students interact with). Finally, it is very relevant the stress of both works regarding the importance of the space in the orchestration of face-to-face activities.

In [4], Dong and Hwang introduce the PLITAZ (Pause Lecture, Instant TutorTutee Match, and Attention Zone) system that minimizes learning progress differences. This work is contextualised by the use of software teaching classes that alternate lecture and practice phases. During the practice phase, two strategies are used to attend students' problems: tutor-tutee match (when a student finishes the practice, she is asked to be a reviewer, and after acceptance, she is commanded to help a peer with problems in this practice) and attention zone (students with problems in the practice surrounded by others with the same problem, should be attended first by the teacher in order to prevent isolation). Therefore, in Dong and Hwang's work the space is also a very important factor. The awareness strategy followed by them is very similar to ours, but their main objective is different because they try to minimize the difference of progress among students and our objective is to enhance orchestration.

Regarding pedagogical concepts relevant to this research, we are going to focus on problem-based learning since it is the methodology used in the lab sessions. Collaborative problem-based learning (PBL), usually considered an active learning methodology [5], is an instructional method commonly used for teaching engineering courses: students are organized in small groups and presented with a challenging problem to solve. In this article, Prince concludes that students do not get better 
assessment results with PBL but they are more motivated and could develop higherlevel skills like information retention, problem solving and critical thinking. Barrows [6] presents a taxonomy for problem-based learning and a set of four educational objectives addressed by PBL, much related to this work: (a) the knowledge about the context; (b) the practice and feedback; (c) self-directed skills; and (d) motivation and challenge.

Finally, the concept of formative assessment is also relevant to our research, since the objective of the interactions in the lab sessions is to provide formative feedback to the students. It is stressed the importance of such feedback for awareness of students [7] and teachers [8].

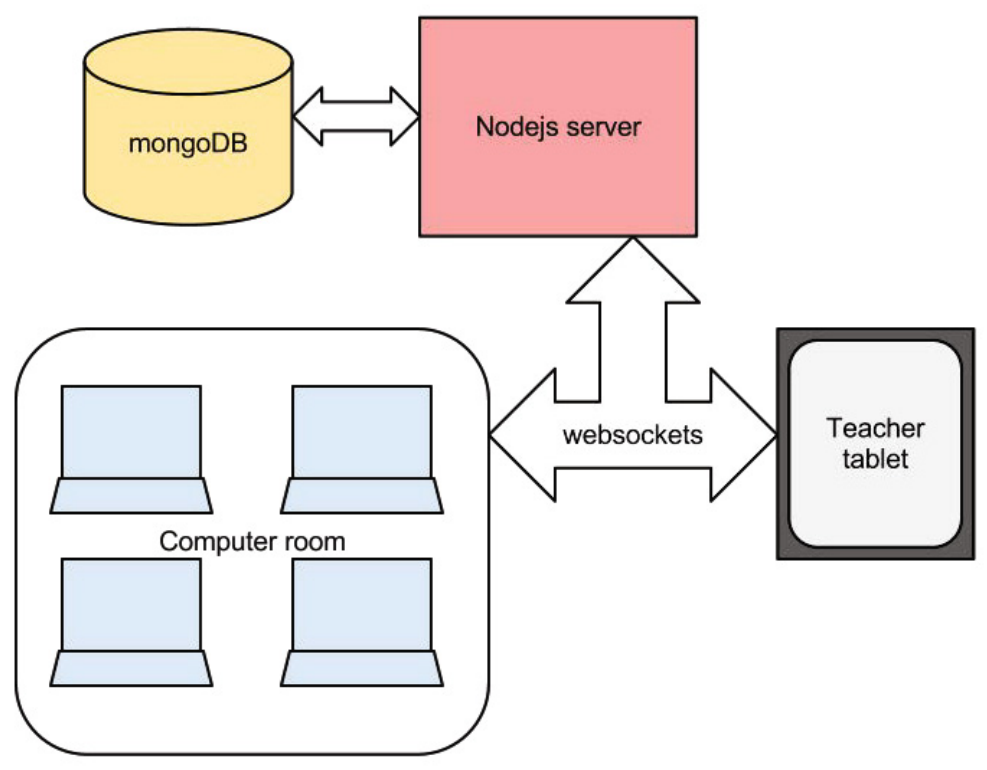

Fig. 1. Awareness system technical architecture

\section{Using Awareness Mechanisms for Orchestration}

As stated before, we are going to make use of the $5+3$ aspects framework described in [2] to classify the different orchestration aspects that we are going to enhance with awareness mechanisms. Four aspects have been identified as part of the orchestration including: the management of the resources in the learning environment; the interventions of the teacher and provision of formative feedback; the collection of evidences for summative assessment; and the re-design of the activity, adjusting some parameters for future enactments. 


\subsection{Awareness System Technical Architecture}

The awareness system we have built assumes the context of problem-based learning in lab sessions, being the assignment of the session delivered to the students as a web page. The system is composed of two parts: one embedded in the web page of the assignment and the other one a tablet web interfaces for the teacher. Regarding technologies (as shown in Figure 1), we have used websockets [9] in order to implement real-time communication of events among students and teachers, as the clients are web browsers. For an easy websocket implementation we used nodejs [10] in the back-end (a server-side JavaScript solution) that is able to manage multiple connections opened with the browsers without performance problems. For the data, we have used mongodb[11], a No-SQL database that uses JavaScript as the script language and JSON as data format. In this way, JavaScript and JSON are used in all the stacks (client, server, database) facilitating the integration of the developed components.

\subsection{Awareness System Interfaces}

In a previous work [12], a exploratory research was presented, consisting of the usage of websocket notifications as a communication backchannel in lab sessions. It also introduced a preliminary version of the students and teacher interfaces. Nevertheless, the design of both interfaces has been changed, taking into account the feedback provided by teachers, students and fellow researchers.

The assignment of the session is a problem-based assignment, composed of several parts or sections. The developed client component for the students (henceforth, student component) analyses the web page of the assignment detecting the sections in the document, and constructs a table of contents. At the beginning of the session, it presents a very simple interface to the students, composed of the following two parts: the main and the aside components.

In the main part of the page (right side of Figure 2), the first section of the assignment is presented (as the "current section" for the students to start with), as well as the references section if existing (list of theoretical references that could be useful for the students during the session); when the students indicate progress (i.e., finished the current section), the next section of the assignment is presented.

On the left side of the screen (aside) there is a fixed part divided in three main regions: (1) in the top: a table of content for the assignment, containing all the sections in the assignment and little circle indicating their status (green: completed, amber: in progress, grey: not initiated); the status circle is clickable for indicating progress (on the current section when it is finished, and on the last finished to undo the progress); at the bottom of this region there is a progress bar indicating the progress of the students in the assignment; (2) in the middle: a red button used to ask a question to the teacher (equivalent to raise hand); when help is requested, the students is prompted to describe the question and the button turns blue; now, the button can be used to indicate that the doubt has been solved (by the teacher or the students themselves) and the student is prompted to describe the answer to her solved question; the position in the queue is also shown to the student because this information is known when raising hand (since you can observe the other students 
that raised hand too); (3) in the bottom: the name of the students working in the PC; when accessing to the assignment, the first thing they have to do is entering their student id; in this part there is also a button to change the students in case it was necessary.

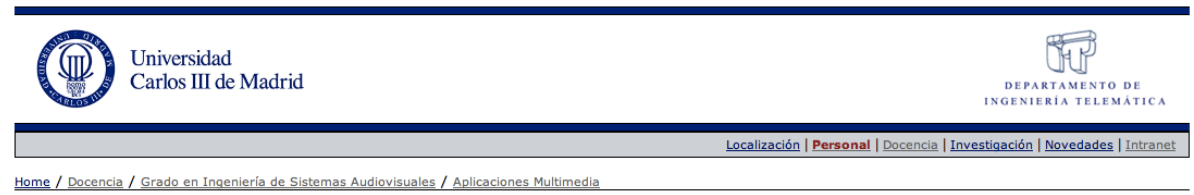

\section{Práctica 3: Video en HTML 5}

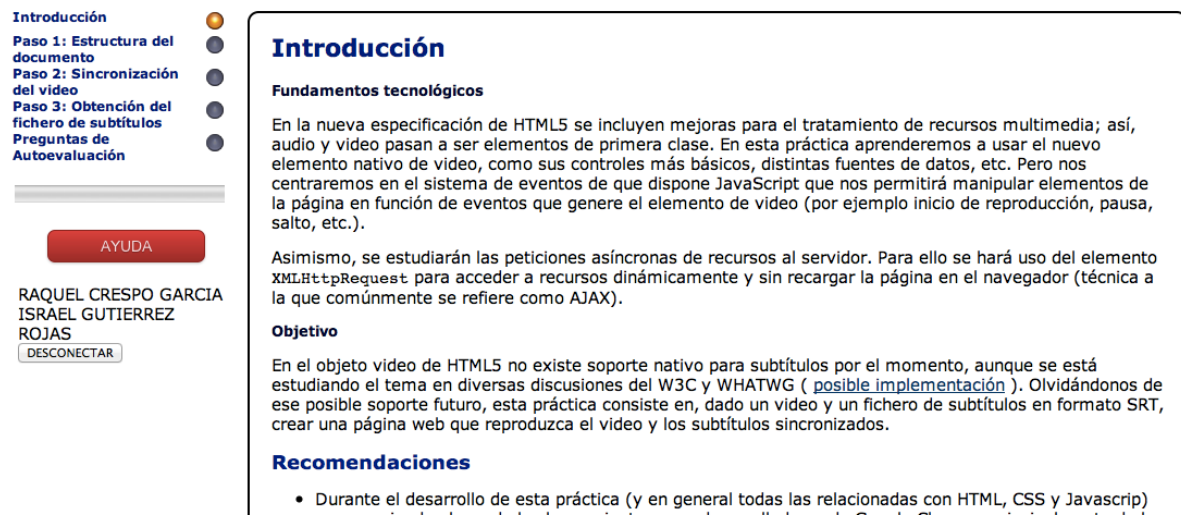

Fig. 2. Students assignment interface

Regarding the tablet web interface for the teacher, it is composed of two parts. The first one (general view, shown in Figure 3) is a representation of the physical classroom where the lab session is carried out. It shows a set of icons representing the PCs in the classroom, which are used as the context for the information of the students working on the PC. In the icons, several types of awareness information are shown. Firstly the background colour of the icon indicates: grey, PC not in use; blue, students working in the PC; or orange to red, the students in the PC asked for help (the colour starts being orange and turns gradually into red in 10 minutes). Secondly, the number in the middle of the icon indicates the current section of the assignment for the students in that PC. Finally, a square in red around the icon of the PC, indicates that these are the students that have been waiting for longer time.

There are also two indicators on the top of the screen: the one on the top-left corner indicates informs about the state of the connection to the server (red: not connected, green: connected); the one on the top-right corner indicates that the teacher has some students waiting for help (red-BUSY: indicates that some students asked for help, green-FREE: indicates that there are no students waiting for help). 


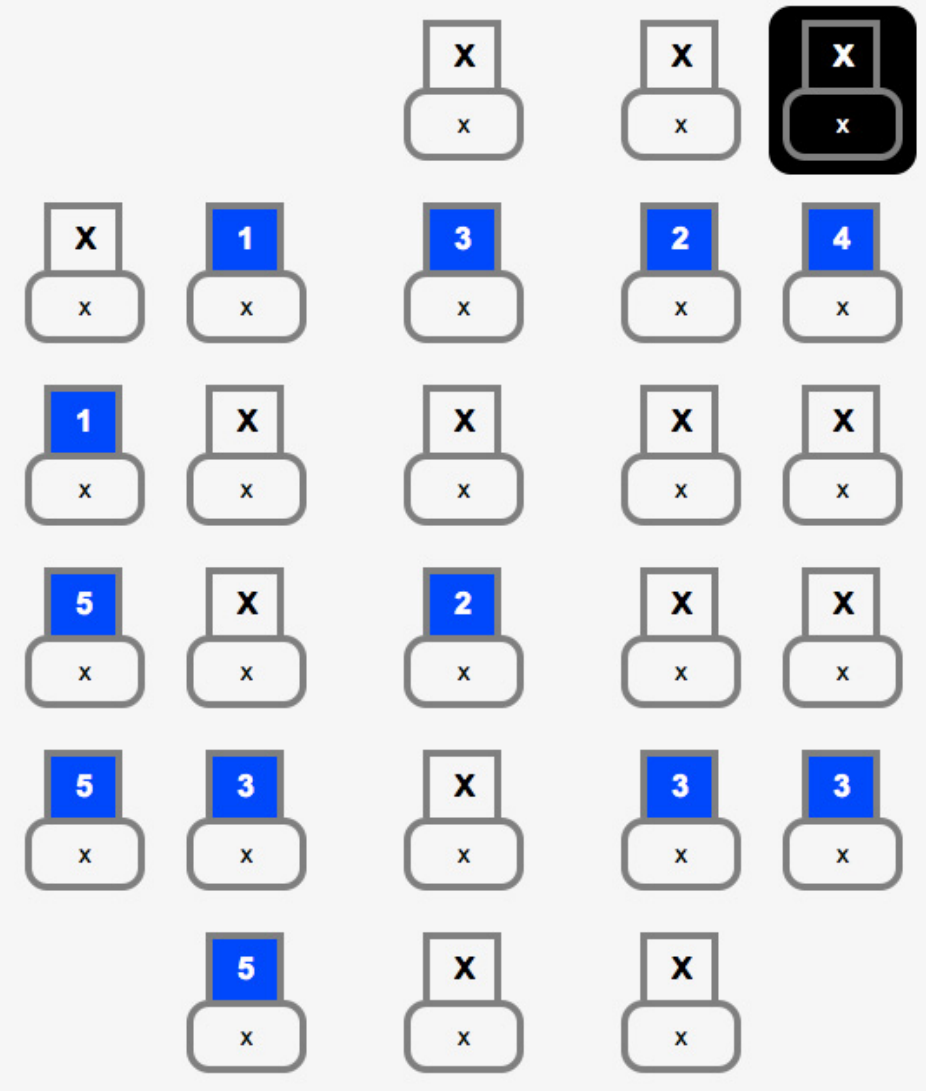

Fig. 3. Teacher interface: general view

The detailed view (shown in Figure 4) appears in the tablet interface when the teacher touches the icon of a PC that is being used by students. In this view, the pictures and names of the students are shown as well as the description of the last question that they asked. Besides that, there is a timer and a button for the teacher to indicate that she is providing feedback to these students. The timer is used for the teacher to be aware of the time devoted in this feedback interaction. When the teacher finishes her intervention, she pushes the button again to stop the timer (or simply push the back button to return to the general view). 


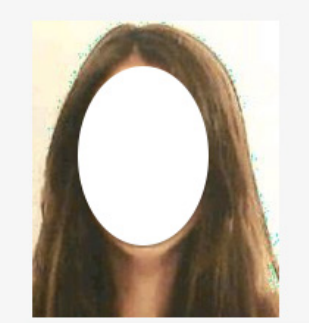

Student 1

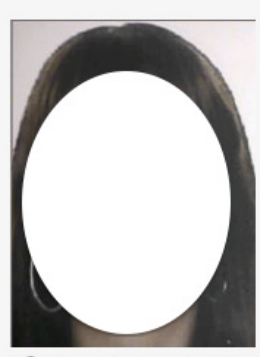

Student 2
Duda: no podemos acceder al elemento boton

00:00

INIT HELP

Fig. 4. Teacher interface: detailed view

\subsection{Awareness System Workflows}

The workflow of the teacher during the session consists of the following steps:

1. connect to the awareness system, indicating the course and session

2. while there is no students waiting for help, she can observe the progress of the students in the general view, and provide feedback to the students proactively

3. when one or more students ask for help, the BUSY/FREE indicator turns red, and a red square indicates the next step (i.e., the students that has been waiting longer for help)

4. when the teacher decides to provide feedback to a group of students, she enters in the detailed view to check the name and pictures of the students and the question they have asked

5. when the feedback starts she presses the button to activate the timer 
6. once the feedback has been provided, she presses the button again and checks the general screen for the next group to be attended

The workflow of the students during the session consists of the following steps:

1. connect to the assignment and enter the student id for the system to identify them

2. start working on the first section of the assignment

3. when they finish a section, indicate progress using the progress indicators (colored circles)

4. when they need feedback of the teacher, push the HELP button and introduce the description of the question

5. when they solve a question (by the teacher or by themselves), push the SOLVED? button and describe the solution to their question

6. if they indicated progress incorrectly, they can use the progress indicator of the last finished section to undo the progress and go back to the previous section

\subsection{Awareness System Benefits}

The benefits of the introduced awareness system for enhancing the orchestration of the lab sessions are presented following the 5+3 aspects framework as defined above. Moreover, the aspects are presented grouped in two categories: aspects used for orchestration during the session (live) and after the session.

- adaptation/flexibility/intervention: during the session, the awareness information about students progress and help is used by the teacher to plan and execute the intervention for feedback provision

- regulation/management: during the session, the awareness information about the time devoted to a group is used by the teacher to manage the timing of the session

- awareness/assessment: after the session, the teacher makes use of the students' progress at the end of the session to determine which ones worked as expected during the session and reward them with a positive grade

- design/planning: after the session, the teacher reviews the questions and answer of the students and plan the future enactment of the activity

\section{Validation of the Awareness System}

The system has been validated in a real setting, a course of Multimedia Applications, in 5 sessions with 4 different teachers ( 2 authors of this work and 2 outsiders). The students in each session ranged from 20 to 30 (10 to 15 groups of 2 students), working in pairs ( 2 students per PC) in the assignment of the lab session. About 800 events were captured, of the following types: 
- connection: students connect to the assignment web page

- finishSection: students indicate a section as completed

- undoFinishSection: students undo the completion of a section

- help: students asked for help

- solved: students indicated that a question have been solved

- initHelp: the teacher starts helping a group of students

- endHelp: the teacher finished the attention to a group of students

In a previous work [13], a set of metrics was presented in order to evaluate an awareness system. One of these metrics is the waiting factor of the lab sessions (ratio between the waiting and the tutoring times). Applying this metric to the collected events, the results obtained were not the expected (see Figure 5).

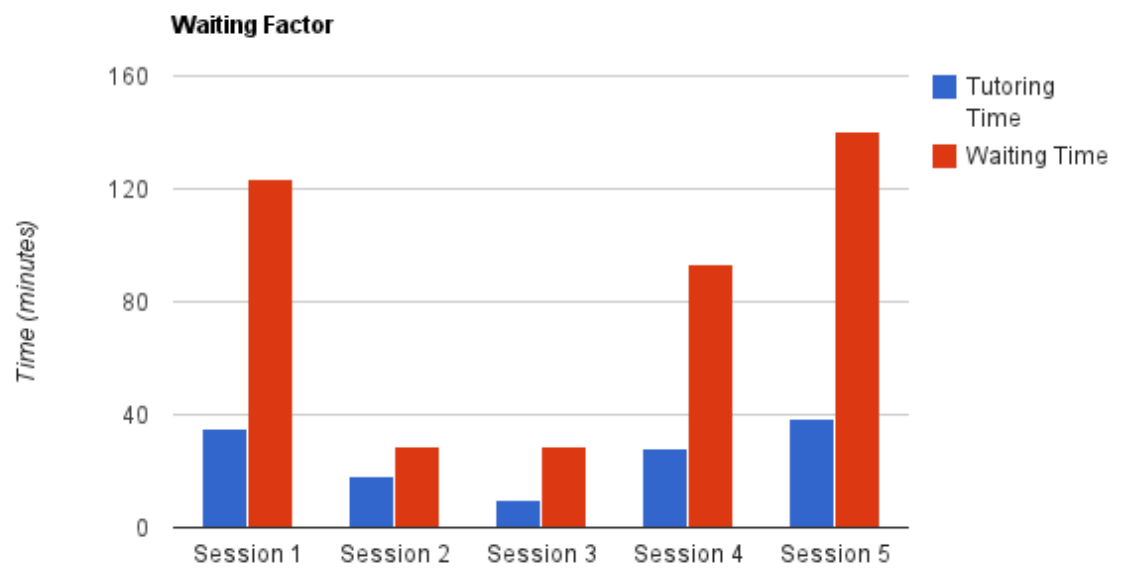

Fig. 5. Tutoring and waiting times per session

The waiting times are always higher than the tutoring times and, therefore, the waiting factor is always greater that 1 . Analysing the data of the sessions (shown in Table 1), we found out that the sessions could be categorised in two: sessions 1 to 4 correspond to a very simple assignments and therefore very few questions arose among the students; instead, session 5 consisted of a complicated assignment and the teacher was solving doubts all along the session (34). The data shows that the waiting factor per se cannot be used to determine the time efficiency of the sessions since the session 5 was very efficient but its waiting factor is the worst. Therefore, a new metric should be defined that combines the measures of time and interventions in order to characterise the efficiency of the session. The definition of such a metric is a future work to this contribution. 
Table 1. Quantitative data collected in Multimedia Applications

\begin{tabular}{|c|c|c|c|c|c|}
\hline $\begin{array}{c}\text { Session } \\
\text { number }\end{array}$ & $\begin{array}{c}\text { Tutoring } \\
\text { time (min.) }\end{array}$ & $\begin{array}{c}\text { Waiting time } \\
\text { (min.) }\end{array}$ & $\begin{array}{c}\text { Waiting } \\
\text { factor }\end{array}$ & $\begin{array}{c}\text { Help } \\
\text { requests }\end{array}$ & $\begin{array}{c}\text { Interven } \\
\text { tions }\end{array}$ \\
\hline 1 & 34.94 & 123.59 & 3.54 & 17 & 17 \\
\hline 2 & 18.13 & 29.11 & 1.61 & 13 & 13 \\
\hline 3 & 9.77 & 28.76 & 2.94 & 14 & 11 \\
\hline 4 & 28.03 & 93.33 & 3.33 & 12 & 12 \\
\hline 5 & 38.34 & 140.46 & 3.66 & 37 & 34 \\
\hline
\end{tabular}

All in all, there could be other factors that conditioned the values obtained. For example, the usage of the system may require a learning curve and thus the parameters could be better in future experiments. Another issue could be that in order to measure the tutoring time, the teachers should press the "INIT HELP" button and, in some occasions, the teachers recognise to have forgotten about doing it.

Nevertheless, besides the quantitative data collected students were asked to fill out a survey about the sessions in which they used the awareness system. The teachers (only the two outsiders) were also interviewed regarding the dynamics of usage of the tool, good/bad features and general feeling.

The main highlight of the interviews are summarised in two good and two bad comments about the systems.

The interviewed teachers identified as the best features of the system that..

- they could find out in a glance which students in the class are working in the session and their progress

- they could be more fair in the distribution of feedback, attending those students who waited longer

They also identified as the worst problems of the system that...

- they did not read the questions of the students in the tool beforehand, but directly asked the students to tell them

- the UI of the teacher interface was improvable, making it adapted to a portable touch device (size of elements, buttons, etc.)

From the students surveys (points in a 1-5 likert scale), it can be stated that the using the tool...

... the tutoring time of the teacher is more fairly distributed (mean 4.38)

... the order of time distribution is more fair (mean 4.46)

... make students concentrate more in solving the problem that in searching for the teacher attention (mean 4.07)

... the students trusts that the teacher is going to help them although they do not raise their hand (mean 4.23 ) 
... the student interface is clear and easy to use (mean 4.00)

... students do not like to write the questions because they prefer to ask her the questions directly

\section{$5 \quad$ Conclusions and Future Work}

In this work, a set of enhancements for learning orchestration in lab sessions based on awareness mechanisms have been presented. The enhancements have been organised following the $5+3$ framework for orchestration, being the following:

- during the lab session, the teacher is informed with the students progress and help requests in order to plan and execute the intervention for feedback provision

- during the session, the teacher is informed about the time devoted to a group in order to manage the timing of the session

- after the session, the teacher makes use of the students' events during the session that may be used as evidences for summative assessment

- after the session, the teacher reviews the questions and answer of the students and plan the future enactment of the activity

The aforementioned enhancements have been validated in a real setting, in a course of Multimedia Applications, demonstrating the effectiveness of the proposed system by means of quantitative (likert scale surveys) and qualitative (interviews) data.

As future work, a lot of lines could be addressed, being the most relevant:

- define metrics that characterise a time efficient system

- integration of formative assessment when completing a section in the assignment: this kind of integration would validate the progress of the students; they would be commanded to deliver a piece of work that proves their progress or do some multiple choice questions for self-assessment

- shared questions: it is a widget for the students to share their questions during the session and they could follow a question of a peer, allowing the teacher to be aware of the most followed questions. A widget based on this principle won the 3rd ROLE widget competition and it is being implemented for the ROLE infrastructure.

- new visualizations of the data collected during the sessions for teacher to better review the session after completion

- provide the students with information about the progress of the class (mean of all students progress) and compare it to her individual progress

- implement new strategies for recommending the next step to the teacher: instead of using always the longer waiting students, different algorithms could be designed following the same principles that the CPU uses for allocating time for processes (FCFS, FJS, Round-Robin)

- a gamification strategy could be implemented for the students to engage more in the session, based on the collected data 
Acknowledgments. This research has been partially supported by the project "Learn3: Towards Learning of the Third Kind" (TIN2008-05163/TSI) of the Spanish "Plan Nacional de I+D+i", the Madrid regional project "eMadrid: Investigación y Desarrollo de tecnologías para el e-learning en la Comunidad de Madrid" (S2009/TIC-1650) and the "EEE project" (TIN2011-28308-C03-01) of the Spanish "Plan Nacional de I+D+i".

\section{References}

[1] Orchestrating learning, Stellar Network of Excellence, http:/ /www. stellarnet. eu/d/1/1/Orchestrating_learning (last accessed April 02, 2012)

[2] Prieto, L.P., Holenko Dlab, M., Gutiérrez, I., Abdulwahed, M., Balid, W.: Orchestrating technology enhanced learning: a literature review and a conceptual framework. International Journal of Technology Enhanced Learning 3(6), 583-598 (2011)

[3] Alavi, H.S., Dillenbourg, P., Kaplan, F.: Distributed Awareness for Class Orchestration. In: Cress, U., Dimitrova, V., Specht, M. (eds.) EC-TEL 2009. LNCS, vol. 5794, pp. 211225. Springer, Heidelberg (2009)

[4] Dong, J.-J., Hwang, W.-Y.: Study to minimize learning progress differences in software learning class using PLITAZ system. Educational Technology Research and Development (243) (2012), doi:10.1007/s11423-012-9233-x

[5] Prince, M.: Does Active Learning Work? A Review of the Research. Journal of Engineering Education 93, 223-231 (2004)

[6] Barrows, H.S.: A taxonomy of problem-based learning methods. Medical Education 20(6), 481-486 (1986), doi:10.1111/j.1365-2923.1986.tb01386.x

[7] Dillenbourg, P., Järvelä, S., Fischer, F.: The Evolution of Research in ComputerSupported Collaborative Learning: from design to orchestration. In: Balacheff, N., Ludvigsen, S., de Jong, T., Lazonder, A., Barnes, S. (eds.) Technology-Enhanced Learning. Springer (2009)

[8] Watts, M.: The orchestration of learning and teaching methods in science education. Canadian Journal of Science, Mathematics and Technology Education (2003), http: / /www. informaworld.com/index/918899916.pdf (retrieved)

[9] Websockets, http://dev.w3 .org/htm15/websockets / (last accessed April 02, 2012)

[10] NodeJS, http: / / nodejs.org (last accessed April 02, 2012)

[11] MongoDB, http: / /mongodb.org (last accessed April 02, 2012)

[12] Gutiérrez Rojas, I., Crespo García, R., Delgado Kloos, C.: Orchestration and Feedback in Lab Sessions: Improvements in Quick Feedback Provision. In: Kloos, C.D., Gillet, D., Crespo García, R.M., Wild, F., Wolpers, M. (eds.) EC-TEL 2011. LNCS, vol. 6964, pp. 424-429. Springer, Heidelberg (2011)

[13] Gutiérrez Rojas, I., Crespo García, R.: Towards efficient provision of feedback in lab sessions. In: Proceeding of the International Conference on Advanced Learning Technologies, ICALT 2012 (accepted, 2012) 